\title{
DIAGNOSTIC CHALLENGES IN A PATIENT WITH COGNITIVE AND MOTOR DISTURBANCES: IS IT HUNTINGTON'S DISEASE?
}

\author{
Stela Atanasova ${ }^{1,2}$, Ivan Dimitrov ${ }^{2,3}$, Sevdzhan Osman ${ }^{2}$, Ara Kaprelyan ${ }^{1,2}$, Kalina Drenska ${ }^{1,2}$ \\ ${ }^{1}$ Department of Neurology and Neuroscience, Faculty of Medicine, \\ Medical University of Varna \\ ${ }^{2}$ First Clinic of Neurology, St. Marina University Hospital, Varna, Bulgaria \\ ${ }^{3}$ Department of Nursing, Sliven Affiliate, Medical University of Varna
}

\begin{abstract}
Huntington's disease (HD) is a fatal genetic disorder that causes the progressive breakdown of nerve cells in the brain. It deteriorates a person's physical and mental abilities usually during their prime working years and there is no cure. HD is known as the quintessential family disease because every child of a parent with HD has a 50\% chance of inheriting the faulty gene. Symptoms usually appear between the ages of 30 to 50 , and worsen over a 10 - to 25 -year period. Ultimately, the weakened individual succumbs to pneumonia, heart failure or other complications. Everyone has the gene that causes HD, but only those that inherit the expansion of the gene will develop HD and perhaps pass it on to each of their children. Every person who inherits the expanded HD gene will eventually develop the disease. Over time, HD affects the individual's ability to reason, walk and speak. Hereby, we describe the case of a 35-year-old patient with HD presenting with cognitive and motor disturbances to underline the diagnostic challenges and differential diagnostic options.
\end{abstract}

Keywords: Huntington's disease, cognitive, motor disturbances

Address for correspondence:

Stela Atanasova

First Clinic of Neurology

St. Marina University Hospital

1 Hristo Smirnenski Blvd

9010 Varna

e-mail: atanasova.g.stela@gmail.com

Received: February 16, 2019

Accepted: March 18, 2019
Huntington's disease (HD) is an inherited genetic disease leading to progressive degeneration of nerve cells in certain areas of the brain. The loss of nerve cells leads to the manifestation of a number of symptoms including uncontrollable movements (chorea), cognitive deficit, emotional disturbances, and a variety of psychiatric symptoms. The onset of symptoms is often in middle age, but the time of manifestation may differ. The mutant protein in HD 
is named huntingtin and results from an expanded CAG repeat (1). It causes neuronal dysfunction and death through disruption of proteostasis, transcription and mitochondrial function and direct toxicity (2). Genetic testing confirms the diagnosis, but mutation gene carriers usually remain asymptomatic for years. Before the clinical manifestations become apparent, alterations in the basal ganglia and cortex are present and can be detected using techniques, such as PET imaging (3). Sometimes the diagnosis can be challenging even in the clinical stage.

A 35-year-old female patient was admitted in the First Clinic of Neurology of St. Marina University Hospital in Varna, Bulgaria, with complaints of frequent forgetfulness and swaying, mostly to the right, for the last 5 years, with gradual progression. She reported that for about 2 years she had had "trembling" of the fingers and toes, and her speech had deteriorated. One year before admission to the hospital, swallowing of solid foods had become difficult, the patient was choking at times, and was experiencing episodes of urinary incontinence. The patient reported no accompanying diseases. Family history included mother with schizophrenia who had died of stroke, uncle who had died at the age of 33 years with memory, speech and motor disturbances, and grandfather who had died at the age of 40 years with similar symptoms. Both uncle and grandfather are on the mother's side. Unfortunately, no medical documents were available.

The neurological examination showed dysarthria and dysphonia, quadripyramidal syndrome with pronounced spastic paraparesis, bilateral dysmetria with mild intention tremor, negative Romberg test, paretic-ataxic gait, paraesthesiae for the palms and soles, urinary incontinence, and bradypsychia. The hematology and chemistry indicators were normal. Serology testing showed positive AntiBorrelia burgdorferi IgM. Brain MRI (Fig. 1) gave evidence of atrophic changes in the cerebellum, pons, parietal and occipital lobes. Cerebellar type multiple system atrophy and spinocerebellar ataxia were suggested as possible differential diagnoses. PET/CT of the brain (Fig. 2) demonstrated severe hypometabolism in both striatums, relatively reduced activity in the pons and suspected reduction of activity in the cerebellum. Echocardiography and indirect laryngoscopy were normal. No Kaiser-Fleischer ring was found on ophthalmological examination. Neuropsychological assessment showed mild memory impairment, decreased verbal fluency and active attention. MMSE score was 27. Anxiety was noted. Finally, genetic analysis was performed. Expanded CAG triplet-repeat on the HTT gene was found, confirming the diagnosis of $\mathrm{HD}$.

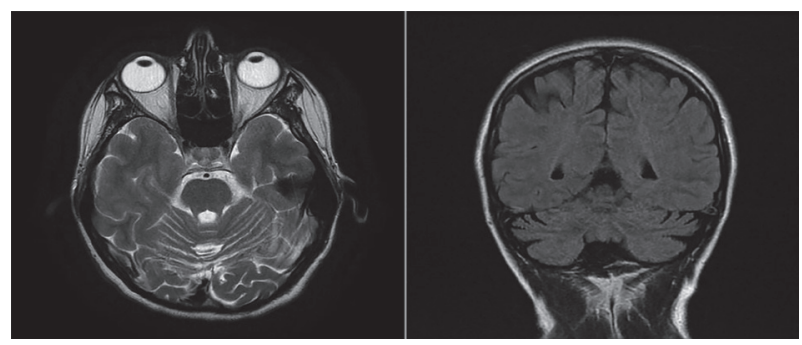

Fig. 1. MRI of the brain, axial and coronal planes

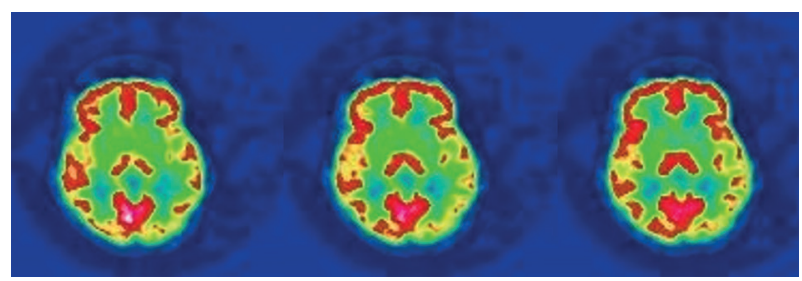

Fig. 2. PET/CT of the brain

On admission of the patient to the Clinic, several possible differential diagnoses were discussed, including CNS infections, multiple sclerosis, Friedreich's disease, and HD. Later, when additional family history data were obtained, attention was focused on HD. Multiple system atrophy was suggested by MRI, but was disregarded because of no match with the history and clinical data. The diagnosis of HD was confirmed on the basis of clinical symptoms, coinciding with family history and genetic analysis. PET imaging was also in line with this diagnosis.

\section{REFERENCES}

1. Walker FO. Huntington's disease. Lancet. 2007; 369(9557):218-28. doi: 10.1016/ S0140-6736(07)60111-1.

2. McColgan P, Tabrizi SJ. Huntington's disease: a clinical review. Eur J Neurol. 2018. 25(1):24-34. doi: 10.1111/ene.13413.

3. Roussakis AA, Piccini P. PET Imaging in Huntington's Disease. J Huntingtons Dis. 2015. 4(4):287-96. doi: 10.3233/JHD-150171. 